\title{
Qualitative Explanation of Phase Shifts Observed in Line Profile Variations
}

\section{H. Montgomery}

IoA, University of Cambridge,UK; e-mail:mikemon@ast.cam.ac.uk

\begin{abstract}
In a series of papers, Telting \& Schrijvers (1997) have demonstrated the correlation between the phase shift across a line, $\Delta \Psi_{0}$, and the $\ell$-value of a mode. In addition, they found that the phase shift of the harmonic (i.e., at frequency $2 \omega$ ) was an indicator of $m$. In this poster, we present a heuristic derivation of these results under a set of idealized assumptions. Within this framework, exact analytical formulae for the $I_{\ell m}(x)$ were calculated, which are available upon request.
\end{abstract}

\section{Simplifying assumptions}

We assume that radial motions dominate horizontal ones (i.e., high-order $p$ modes), that the natural width of the lines is much less than the rotational broadening, that line profile variations are produced only by the Doppler shifting of surface elements (e.g., changes in the intrinsic profile shape are ignored), that the effect of rotation on the eigenfunctions is negligible, and that the pulsational velocity is small compared to $V_{e} \sin i$. The line profile is then given by

$$
I(x, t)=\iint d x^{\prime} d y^{\prime} b f\left(x-x^{\prime}-v_{p}\right),
$$

where $x$ is the horizontal position in the profile with respect to the center of the line (i.e., the observed wavelength in units of $\left.V_{e} \sin i\right), f(x)$ is the intrinsic shape of the line profile, $v_{p}=v_{p}\left(t, x^{\prime}, y^{\prime}\right)$ is the pulsation velocity projected along the line of sight, $x^{\prime}$ and $y^{\prime}$ are the horizontal and vertical coordinates in the projected disc of the star (in units of $V_{e} \sin i$ ), and $b=b\left(x^{\prime}, y^{\prime}\right)$ is the limb darkening.

If we expand this expression for small $v_{p}$, assume an arbitrarily thin intrinsic profile, and assume that $v_{p}$ is given by linear pulsation theory, i.e.,

$$
f\left(x-x^{\prime}\right)=\delta\left(x-x^{\prime}\right), \quad v_{p}(t, x, y)=\operatorname{Re}\left[e^{i \omega t} \tilde{v}_{p}(x, y)\right]
$$

then we obtain

$$
I(t, x) \equiv \overline{I(x)}+I_{0}(t, x)+I_{1}(t, x)+O\left(v_{p}^{3}\right)
$$

where $\overline{I(x)}$ contains the time independent part of the line profile, and

$$
\begin{aligned}
& I_{0}(t, x)=\operatorname{Re}\left[e^{i \omega t} I_{\ell m}^{(0)}(x, \theta)\right], \quad I_{\ell m}^{(0)}(x, \theta) \equiv \frac{\partial}{\partial x} \int_{-y_{0}}^{y_{0}} d y b(x, y) \tilde{v}_{p}(x, y) \\
& I_{1}(t, x)=\operatorname{Re}\left[e^{i 2 \omega t} I_{\ell m}^{(1)}(x, \theta)\right], \quad I_{\ell m}^{(1)}(x, \theta) \equiv \frac{1}{2} \frac{\partial^{2}}{\partial x^{2}} \int_{-y_{0}}^{y_{0}} d y b(x, y) \tilde{v}_{p}^{2}(x, y)
\end{aligned}
$$


where $y_{0} \equiv \sqrt{1-x^{2}}$. Clearly, the $\left\{I_{\ell m}^{(j)}\right\}$ contain all the spatial/wavelength information concerning the line profile variations, accurate to $O\left(v_{p}^{3}\right)$.

\section{Qualitative derivation of phase shifts}

We consider modes with inclination angles which are fairly equator on $(\theta \sim 60$ $90^{\circ}$ ), since this is the statistically more likely case. Away from the edge of the disk, we have approximately $x \approx \sin \phi \approx \phi$, with $y \approx \cos \vartheta$, so we may write

$$
\tilde{v}_{p}(x, y) \approx \mu(x, y) P_{\ell m}(\cos \vartheta) e^{i m \phi} \approx \mu(x, y) P_{\ell m}(y) e^{i m x}
$$

where $P_{\ell m}(y)$ is the associated Legendre polynomial, and $\mu(x, y)=1-\sqrt{x^{2}+y^{2}}$. Assuming that the spatial variations of $\tilde{v}_{p}$ are much more rapid than those in the limb darkening and $\mu$, we find

$$
\begin{aligned}
I_{\ell m}^{(0)}(x, \theta) & \approx \frac{\partial}{\partial x} \int_{-y_{0}}^{y_{0}} d y b \tilde{v}_{p} \approx\left(\frac{\partial}{\partial \phi} e^{i m \phi}\right) \int_{-y_{0}}^{y_{0}} d y \mu b P_{\ell m}(y) \\
& \propto e^{i m \phi} \int_{-y_{0}}^{y_{0}} d y \mu(x, y) b(x, y) P_{\ell m}(y) .
\end{aligned}
$$

As we move across the profile from left to right $(x=-1$ to +1$), \phi$ changes by $\pi$, leading to a complex phase change of $|m| \pi$. In addition, the limits over which we are integrating $P_{\ell m}(y)$ change, since $y_{0}=y_{0}(x)$. The function $P_{\ell m}(y)$ has $\ell-|m|$ nodes for $y \in[-1,1]$, so the changing weighting of the positive and negative regions can lead to up to $\ell-|m|$ sign changes. Each sign change is equivalent to a phase shift of $\pi$, so the total phase shift across the line is

$$
\Delta \Psi_{0}=|m| \pi+(\ell-|m|) \pi=\ell \pi
$$

Repeating this analysis for variations at the harmonic, we find that

$$
I_{\ell m}^{(1)}(x, \theta) \propto e^{i 2 m \phi} \int_{-y_{0}}^{y_{0}} d y \mu^{2}(x, y) b(x, y) P_{\ell m}^{2}(y)
$$

Since the quantity $P_{\ell m}^{2}(y)$ does not undergo sign changes, the only phase change across the profile is given by the complex phase factor, so we have $\Delta \Psi_{1}=2|m| \pi$.

These results are supported by and consistent with those obtained by direct numerical integration by Telting \& Schrijvers (1997), as we summarize below:

\begin{tabular}{c|c} 
Telting \& Schrijvers (1997) & \multicolumn{2}{|c}{ analytical derivation } \\
\hline$\ell \approx 0.10+1.09\left|\Delta \Psi_{0}\right| / \pi$ & $\ell \approx 1.00\left|\Delta \Psi_{0}\right| / \pi$ \\
$|m| \approx-1.33+0.54\left|\Delta \Psi_{1}\right| / \pi$ & $|m| \approx 0.50\left|\Delta \Psi_{1}\right| / \pi$
\end{tabular}

Thus, we have given a heuristic derivation of the relationship between the total phase shift across across a line, measured at the mode frequency and its first harmonic, and the $\ell$ and $m$ values of the mode. Most significantly, we can explain the result that the phase shift at the mode frequency is mainly a function of $\ell$, while the phase shift at the harmonic is predominantly a function of $m$.

\section{References}

Telting, J. H. \& Schrijvers, C. 1997, A\&A, 317, 723 